\title{
Essai sur une thanatocratie islamique. Le cas des combattants suicidaires arabo-musulmans
}

\section{Bruno Etienne}

\section{(2) OpenEdition \\ 1 Journals}

\section{Édition électronique}

URL : http://journals.openedition.org/conflits/2099

DOI : $10.4000 /$ conflits.2099

ISSN : 1777-5345

Éditeur :

CCLS - Centre d'études sur les conflits lilberté et sécurité, L'Harmattan

\section{Édition imprimée}

Date de publication : 1 novembre 2006

Pagination : 47-61

ISBN : 2-296-01436-4

ISSN : 1157-996X

\section{Référence électronique}

Bruno Etienne, «Essai sur une thanatocratie islamique. Le cas des combattants suicidaires arabomusulmans ", Cultures \& Conflits [En ligne], 63 | automne 2006, mis en ligne le 09 novembre 2006, consulté le 30 mars 2021. URL : http://journals.openedition.org/conflits/2099 ; DOI : https://doi.org/ $10.4000 /$ conflits.2099

Ce document a été généré automatiquement le 30 mars 2021.

Creative Commons License 


\title{
Essai sur une thanatocratie islamique. Le cas des combattants suicidaires arabo-musulmans
}

\author{
Bruno Etienne
}

1 Une grande partie de la société dite "occidentale » est émotionnellement bouleversée par le terrorisme islamiste, souvent confondu avec l'islam tout court. Le recours à des modes non convenus de violence politique a pourtant été chose courante en Europe et dans les Amériques, mais peu de nos compatriotes semblent faire la liaison. Par exemple, se pose-t-on la question de savoir si la Révolution française a fait plus ou moins de victimes que la Révolution iranienne? Est-ce que les terroristes sont des résistants? Tout le monde a oublié que le «colonel» Fabien a exécuté/assassiné un officier allemand dans le métro parisien et que la répression qui a suivi a coûté la vie à plus de cent de ses camarades du «parti des fusillés». Sans la violence qui a fait des «victimes innocentes ", l'Algérie serait-elle indépendante? Y aurait-il d'un côté une violence et des attentats légitimes pour la simple raison qu'ils sont nationalistes et, de l'autre, une résistance illégitime, et donc terroriste, à partir du moment où elle ne suit pas les règles et les balises du consensus des « nations civilisées »?

2 Il s'agit donc, à l'encontre des préjugés ambiants, d'essayer de comprendre et d'expliquer cette forme extrême de violence que revêt le combat suicidaire.

La pulsion de mort : combattre la mort en la donnant

3 La violence primitive, si l'on peut dire, est une pulsion primaire, présente chez l'animal et chez les tout jeunes enfants, ainsi que chez certains sujets adultes, soit qu'ils se trouvent placés dans des conditions particulières, soit qu'ils n'aient pas "grandi». Cette pulsion correspond à une légitime défense aux yeux de celui qui l'enclenche sans intention, la cible de cette violence «naturelle » et pulsionnelle étant indifférente à celui qui la déploie. Tel n'est pas le cas de l'attentat contemporain dit "kamikaze ${ }^{1}$ ", lequel n'est pas une violence de ce type (infantile) puisqu'il a une cible précise, que ce soit l'Israélien, le Juif, l'occupant et/ou son comparse occidental. Ne serait-il que le 
signe ou le symptôme d'une sensibilité frustrée ? Ou, comme un certain essentialisme le suggérerait, l'expression d'une agressivité arabo-islamique « naturelle »?

4 L'agressivité, telle que la définit par exemple Jean Bergeret ${ }^{2}$, serait une composante de la personnalité secondaire, donc plus tardive, en lien avec l'érotisation de l'œEdipe où l'intégration de la violence primaire s'est pervertie en s'associant à la notion de plaisir dans un tableau de bases narcissiques fragilisées. C'est une piste intéressante que ceux - anthropologues ou psychiatres - qui travaillent sur la misère sexuelle des jeunes arabes urbanisés et frustrés, devraient explorer plus avant ${ }^{3}$. Mais on ne saurait en tirer de trop simples conclusions. Nous verrons certes plus loin que l'aspect narcissique est bien survalorisé dans le cas des combattants palestiniens, mais nous n'avons pas d'enquêtes suffisantes pour lier leur décision à leur sexualité.

5 Le passage à l'acte, le passage à la violence n'est pas le fait du seul petit enfant qui sommeille en nous. Si, «normalement ", la violence et l'agressivité sont socialisées/ canalisées par l'éducation et peuvent même être utilisées positivement, c'est qu'elles permettent l'autoconservation de l'individu, une certaine emprise sur l'environnement, et qu'elles construisent l'estime de soi. Que se passe-t-il donc quand elles (l'une ou l'autre, l'une et l'autre) entraînent un individu à commettre un acte irrémédiable, souvent contraire aux valeurs mêmes de la société à laquelle cet individu adhère et dont il intériorise les valeurs? Autrement dit : à partir de quel moment la pulsion de mort l'emporte-t-elle sur l'Eros?

6 La pulsion de mort, qui apparait comme concept chez Sigmund Freud en 1920 dans Audelà du principe de plaisir ${ }^{4}$ à partir de l'étude de certains traumatismes subis par les combattants de la guerre de 1914-1918, a suscité beaucoup de résistances et de réticences. Au moins savons-nous que cette pulsion n'est pas l'agressivité ${ }^{5}$, laquelle est plutôt à ranger dans la pulsion de vie ; mais ceci est discuté, car certains auteurs voient dans la pulsion d'agressivité une sorte de pulsion de mort tournée vers l'extérieur. Elle ne relève pas non plus du sadisme ou du masochisme, qui sont des pulsions agressives exercées vers l'extérieur ou vers soi-même. Elle est peut-être une dialectique entre les pulsions actives, passives et d'échange : liaison/déliaison, un phénomène d'apoptose ${ }^{6}$ en quelque sorte, à rapprocher dans le champ religieux du désir de nirvâna bouddhiste ou du fana des mystiques musulmans, qui amènent un système psychique vers sa désorganisation. Etant donné les circonstances envisagées ici, la même force de déliaison pousserait l'acteur à produire un acte irréversible, chargé, pour l'édification de l'environnement social, de significations positives.

Opération suicide et société

7 La plupart des Etats-nations trouvent «normal » et même glorieux le sacrifice de leurs enfants morts pour la patrie. Ils les honorent et les pleurent après la victoire ou la défaite. En revanche, les nations et les "nations civilisées " semblent horrifiées par le sacrifice volontaire et les combattants suicidaires du type "kamikaze ${ }^{7}$ " au-delà des victimes innocentes de ces "attentats", alors que les mêmes ne manifestent guère d'états d'âme au vu des bombardements des populations civiles arabes qui durent depuis plusieurs décennies et qui sont pieusement nommés « frappes chirurgicales » et " dégâts collatéraux »...

8 Tout en étant fort éloigné du modèle japonais, l'exemple arabo-musulman actuel suscite une horreur non feinte, mais qui s'en tient à l'émotionnel, sans que jamais, ou rarement, l'analyse aille très loin quant aux motivations, aux conditions objectives de production et à la culture de «terroristes » qui se considèrent comme des résistants. 
Des hommes et des femmes se sacrifient pour une cause, en se légitimant eux-mêmes par un référentiel plus souvent religieux que politique. Le narcissisme n'est pas absent de leur acte; dans ce cas-ci, on a une identification héroïque avec le moi idéal archaïque. Mais on ne saurait oublier la logique de l'honneur des sociétés tribalopatriarcales, ni la déréliction des muztadhafin ("déprivés»), terme utilisé pour désigner tous les frustrés de la modernité, les laissés-pour-compte. Enfin, il n'est pas possible de faire l'impasse sur l'histoire dramatique de réfugiés dans leur propre pays, qui n'ont de statut ni national ni étatique.

9 L'imaginaire islamique se constitue - comme celui des autres peuples ou religions - à partir de structures anthropologiques archaïques, lointaines et proches, historiques et mythiques, et de traditions utilisées par excès de modernité. C'est sur ce terreau que s'est développé l'islam politique. La mort du président égyptien Nasser en 1970 marque la date symbolique des débuts de celui-ci. Les guerres perdues contre Israël - la Guerre des six jours en 1967 et la Guerre du Kippour en 1973 - en ont été l'accélérateur. Par ailleurs, tous les pays arabes, depuis le partage de l'Empire ottoman, ont fait l'expérience de la dictature. Ils se sont dotés de régimes militaires ou policiers qui ont encore aggravé le sort de leurs peuples frustrés d'une véritable démocratisation, tandis que le détournement de la rente pétrolière créait de criantes inégalités. Ils ont eu largement recours à la répression musclée de leurs opposants au point qu'il est possible d'établir une relation entre la violence de l'Etat et celle des « terroristes » islamistes ou autres.

10 C'est le déni de cet échec historique généralisé qui a renforcé la tyrannie du Bien ${ }^{8}$ et élevé le meurtre et le suicide ${ }^{9}$ au rang de sacrifice comme dernier rempart de l'aporie suprême : l'impasse dans laquelle s'est trouvé le discours nationaliste progressiste et identitaire arabe comme avatar du «discours du maître » et son double, le discours capitalistique des élites pétrolières ou celui des clercs musulmans stipendiés. Celui-ci est bien connu. En revanche, nous reviendrons sur la glorification du passé utilisée par les théoriciens du jihad actuel et dans lequel les radicaux de tous bords font entrer la violence suicidaire islamique qui nous intéresse ici.

11 Sans doute conviendrait-il, si la place ne nous manquait, de préciser les termes employés tant par les acteurs que par les observateurs. Au lieu du très médiatique "kamikaze ", les Palestiniens utilisent des termes renvoyant à une autre culture du combat et de la mort : les terroristes sont des combattants mujahid, fidaï, chahîd et même moussabbal, et donc des résistants. Il ne nous faudrait pas moins préciser le statut de la mort dans la culture monothéiste et dans l'islam, ainsi que le statut du suicide, et enfin confronter le sacrifice politique national/patriotique et/ou religieux avec ce que la psychanalyse nous enseigne sur la pulsion de mort et l'ethnopsychiatrie sur la thanatocratie - le gouvernement de la mort dans une société -, non sans se référer à René Girard, pour qui le sacrifice rituel protège l'homme de la violence ${ }^{10}$.

12 Combattre la mort en la donnant? L'aporie n'est qu'apparente ${ }^{11}$. La pulsion de mort résulte du trop-plein d'énergies rendues libres par l'échec des capacités de représentation d'un individu : quand il n'y a plus rien, ni modèle politique, ni utopie, ni espoir, ni solution, et donc que les représentations du possible s'arrêtent, on éclate ! Ce trop-plein d'excitation entraîne une rupture : l'acteur, ou l'agent comme dirait Pierre Bourdieu, est vidé de ses propres désirs. Il est alors l'objet d'un mouvement de déliaison dont la névrose de guerre est l'exutoire. «Cette forme démoniaque dont l'essence est 
bien la destruction: le mal qui est en même temps force créatrice puisque de la destruction de deux individus [le tuant et le tué] naît un nouvel individu ».

Mais à quel moment un acteur - manipulé ou pas - passe-t-il à l'acte d'autodestruction? Et dans quelles conditions «objectives » une société tout entière devient-elle «folle » au point de massacrer ses propres enfants, comme ce fut le cas en Algérie dans les années 1990 où, faute de l'Autre, juif ou européen, à massacrer, il restait le frère différent ? Pleurer sur son passé ne suffisant plus, la haine de soi allait se transformer en haine de l'Autre ${ }^{12}$.

Archéologie « religieuse » : ontogénèse du suicide

Dans la mesure où l'islam fait partie intégrante de la révélation monothéiste et que le Coran lui-même renvoie plus de cent fois aux textes précédents et aux prophètes (la Bible, y compris les Evangiles), le statut de la vie, de la mort et du suicide est identique dans les trois religions monothéistes : Dieu donne la vie, qui est sacrée, et la reprend quand il le veut ${ }^{13}$; le suicide est rigoureusement interdit, ainsi que le meurtre, qui est strictement « réglementé » aussi bien dans la « loi du talion ${ }^{14}$ », que dans la « dette du sang ${ }^{15}$ ».

Bien entendu, dans la réalité, les choses sont à la fois plus compliquées et plus subtiles, et la jurisprudence est immense sur ces sujets. Dans une perspective eschatologique, la finitude de l'homme n'induit pas sa finalité : accomplir le dessein du Créateur. Certains sont tentés d'accélérer le processus afin qu'advienne le royaume de Dieu: le monde pécheur doit être détruit; plus proche est l'Apocalypse, plus proche est le retour du Messie (le Christ et Ali pour certains Chiites). Pour échapper à la dégradation entropique de l'Univers, mourir et faire mourir valent la peine.

Celui qui se sacrifie passe dans l'univers du sacré au prix d'une mort violente, mais celle-ci change son univers, qui se voit à son tour imprégné d'éternité. Par cet acte, il régénère le temps du devenir, en l'occurrence celui de sa propre mort et son espace, les limites oppressantes du monde profane.

Le chahîd, témoin-martyr, devient, selon le mot de Maccoby Hyam, un "exécuteur sacré ». En ce sens, le chahîd est ontologiquement différent du «kamikaze » qui, lui, appartient à une société sans "Dieu», où les cycles de vies ne s'arrêtent que si l'on atteint le nirvâna en mettant fin à la loi du dharma ou, plus exactement, à la fatalité du karma. C'est alors pour lui la libération par le vide, et non la plénitude divine qui est la récompense suprême.

Dans le cas particulier du tyrannicide ${ }^{16}$ islamique par attentat-suicide, en application de la « sixième obligation absente », le combattant suicidaire revêt, selon les points de vue, les traits du fanatique, du serviteur zélé de la loi inflexible, de l'utopiste acharné, du sicaire assassin - assas wa hashshash, gardien du Temple et mâcheur de haschisch. Emanation de la transcendance, animé par l'impatiente espérance d'un autre monde, il est a priori exclu de celui-ci. Convoquant Dieu, il le fait descendre, le prend à témoin, le mobilise au profit de la communauté de ceux qui attendent l'écrasement de cette terre peccamineuse par les nuées venues du Ciel, et le Rédempteur triomphera alors de la Bête. Et "ceux qui ont témoigné » seront assis à la droite du Père... Tel est donc le chahîd. Ce n'est pas sa main qui a tué, ce que le Coran affirme plusieurs fois ${ }^{17}$, puisqu'il s'est aboli en tant que sujet jusqu'à compter pour inutile son passage terrestre, comme il tient pour nulle la vie de ceux qui refusent le Message authentique. La mort de l'autre - le kafîr, l'impie, l'infidèle (à l'exclusion des "gens du livre », chrétiens et juifs), et jusqu'au Musulman tiède - est propitiatoire et expiatoire. La route de Jérusalem passe 
par La Mecque ! L'ennemi proche qui entretient la fitna ${ }^{18}$, ce désordre doctrinal auquel plusieurs versets font allusion comme mal suprême qui détruit l'Umma, la "communauté", littéralement une "matrie», est encore plus insupportable que l'ennemi lointain, celui qui apporte un soutien extérieur aux mauvais Musulmans comme par exemple les Etats-Unis à l'Arabie saoudite.

Le combattant palestinien n'est pas dans cette situation, lui qui affronte la puissante armée israélienne dans une guerre asymétrique. Les ennemis sont de force inégale. De plus, le Palestinien se sent l'objet du mépris de ses «frères » arabes nantis qui lui font la charité verbale et de celui des Israéliens avec le Mur, les check-points et le reste ${ }^{19}$. Mais le mépris va plus loin encore, ainsi qu'il ressort du documentaire israélien de Yoav Shamir ${ }^{20}$ dans lequel un soldat israélien, oublieux sans doute de ce que les nazis pensaient de ses ancêtres martyrisés, déclare : "Les Palestiniens sont des animaux, ce ne sont pas des hommes ». Les usines à fabriquer le mépris et la haine transforment alors la haine de soi, latente, en haine de l'Autre. Absolue.

Exclure l'altérité en accomplissant des meurtres purificateurs implique cependant qu'on se sente agressé de toutes parts. Cette fermeture paranoïaque tient au fait que tout idéal du "moi » est confondu avec un «nous » idéal imaginaire islamique dans l'Unité absolue, la Tawhîd, l'» Unicité » : celle de Dieu qui induit celle de l'Umma, la fusion dans l'Un. La mise en avant de ce monisme existentiel imaginaire donne l'illusion de se saisir de la totalité de l'être musulman accompli par un faire musulman... islamiste. Il s'agit bien en effet de relire ce discours de légitimation comme " si », "signifiant maître », selon Lacan ${ }^{21}$, un discours abusivement légitimé par une référence au transcendantal et à l'inimitabilité du texte fondateur, donc sans interprétation possible hors du canon : mais qui est légitime pour dire la doxa?

Qui, en particulier, dans l'islam acéphale, saurait dire si tel acte individuel référé à des préceptes coraniques contradictoires ${ }^{22}$, répond à une doxa qui n'a que les apparences $\mathrm{du}$ consensus et qu'apparemment, le suicide de combat prend au dépourvu? La proclamation du jihâd permet-elle au moins de déblayer les incertitudes qui pèsent sur la qualification religieuse du «kamikazat»? Encore faudrait-il qu'elle émane d'autorités légitimes dans des circonstances appropriées, ce qui, notons-le, exclut d'emblée les fatwa intempestives d'» émirs » ou de théologiens autoproclamés, plus ou moins liés à un néo-salafisme proche lui-même de l'intégrisme wahabite saoudien. La polysémie du jihâd, au demeurant, qu'a remarquablement déchiffrée Jean-Paul Charnay dans son ouvrage de référence Principes de stratégie arabe, n'aide pas à y voir clair ${ }^{23}$. Elle permet au moins de distinguer entre les combattants de type palestinien nationalistes et frustrés, et les «kamikazes » d'Al-Qaïda, internationalistes et nantis, voire occidentalisés; les "terroristes islamistes locaux ", type GIA, étant d'un genre à part, plutôt nationalistes ${ }^{24}$. La différence en tout cas est manifeste dans le discours interne entre al-'umma al-islamiya et al-watan al-'arabiya, entre la communauté musulmane comme « matrie » et la patrie arabe.

Les tenants de la première sont plutôt des « délocalisés ». Ceux qui ont sévi à New York, à Madrid où à Londres passent plutôt pour des nantis culturels immergés dans les sociétés d'accueil et vivant "à l'occidentale». Dans leurs attentats, qui visent les ennemis de l'islam tel que le conçoit la nébuleuse Al-Qaïda, le référent "religieux » ne s'impose pas clairement, à cela près que le terme de « croisé » est utilisé pour désigner cet ennemi à la fois proche et lointain. Il en va autrement des attentats locaux comme ceux d'Egypte, ou d'Arabie saoudite qui visent l'ennemi proche, selon la thèse de G. 
Kepel, c'est-à-dire les gouvernements arabo-musulmans "impies » et, au-delà, leurs alliés occidentaux.

On notera au passage qu'en dépit d'une propagande considérable des Gardiens de la révolution iraniens, il ne semble pas que la « martyrophilie » chiite ait eu une influence décisive sur les acteurs aujourd'hui les plus en vue, palestiniens, "terroristes sunnites ", et surtout pas sur les candidats « européens » de nos banlieues qui agissent plutôt par solidarité dans leur groupe de pairs, même si certains recruteurs viennent de l'extérieur. Dans tous les cas enfin, les femmes y sont assez rares et, évidemment, largement mises en scène ${ }^{25}$.

Les suicidants

Délocalisés ou non, quelques instants avant leur attentat, les futurs chouhada ${ }^{26}$ téléphonent à leur mère pour leur dire: "Maman, je vais aller au paradis, je suis prêt »; « je vais aller à la place qui m'est réservée », ce qui correspond à un verset cité plus haut. Certains se considèrent comme déjà morts, « en mission divine sur la Terre et sur le point d'être appelés par le Seigneur ", ce qui correspond en effet là aussi à plusieurs versets du Coran qui conseillent de n'être qu'un passager. Parfois, certains demandent que l'on prie pour eux.

Les familles, quant à elles, aussi bien au Maroc qu'en Palestine, se contentent de déclarer : "Dieu l'a pris avec Lui, nous, nous ne savons rien... », alors que, pour les représentants d'Al-Qaïda impliqués dans les attentats du 11 septembre 2001, on a entendu certains parents renier leur progéniture: «Ce n'est pas mon fils qui a fait cela ». Les cérémonies de condoléances sont largement diffusées, parfois en contradiction avec le travail du deuil propre à l'islam populaire : les fatwas légitimant ces actes sont assez alambiquées et produisent des débats très contradictoires. Elles sont toujours prises en référence à la défense de l'islam: "Ils combattent dans le chemin de Dieu, ils tuent et ils sont tués ${ }^{27}$ "

26 A la limite, cela a peu d'importance, car, comme le soulignait Max Weber, il n'est pas nécessaire d'être un virtuose en théologie pour croire et être religionnaire. Dans la grande majorité des cas, les familles des martyrs se glorifient de cet acte et rendent un hommage au mort selon des rituels populaires parfois peu orthodoxes.

27 Les rituels autour du corps du défunt posent aussi problème. Car, le plus souvent, le corps n'est pas récupéré, tout simplement parce qu'Israël ne le rend pas ${ }^{28}$ (même problème, on l'a vu récemment, pour des Israéliens. Or, théoriquement, le corps doit être lavé puis enroulé dans un ou trois, cinq ou sept linceuls ${ }^{29}$ (si l'on compte le turban et la tunique), la dépouille parfumée placée sur le côté droit, le convoi funèbre suivi à pieds. Puis le cadavre est enterré dans la glaise, en référence au fait qu'il fut créé de poussière ${ }^{30}$. "Nous appartenons à Dieu et c'est à Lui que nous revenons " est une formule très courante prononcée en cette occasion. La prière (al-salat al-janza) est elle aussi très précise autour du «tourment de la tombe » ('adab al-qabr) : « Dis : l'ange de la mort auquel tu es confié t'accueillera puis vous serez ramenés vers votre Seigneur ${ }^{31}$ ». Les deux anges de la mort, Nâkir et Munkir ${ }^{32}$ sont convoqués ainsi qu'Azrafil et Azrail ${ }^{33}$.

28 Ce rituel-liturgie ne s'applique théoriquement pas aux suicidés, comme l'atteste un cas où le Prophète refusa de dire « la prière pour l'absent » (al-salat al-mayyat) sur le corps d'un chahîd qui s'était précipité dans la mort. Mais on connaît aussi d'autres hadiths qui ont montré une attitude contraire. Le grand jurisconsulte Mawardi, mort en $1058^{34}$ rapporte des faits et paroles en ce sens lors de la célèbre bataille de Badr ${ }^{35}$. Les 
puritains récusent les lamentations comme conduite païenne, mais la pratique des pleureuses est très courante dans l'islam populaire, et certains " dicts » (hadith) ou traditions indiquent même la hiérarchie de celles-ci : la mère et les soeurs puis les autres.

Nous trouvons cependant dans l'hagiographie musulmane et dans le corpus de hadiths, aussi bien que dans les Sirat ${ }^{36}$, des indications à propos de ce qu'on peut regarder comme une exception à ces rituels. Certains Musulmans font donc, en ce sens, référence à une parole du Prophète lors de la bataille de Ohod (ou Uhud), en 625, au cours de laquelle le Prophète est blessé. La Sunna (la «tradition») rapporte plusieurs hadiths reproduisant les propos tenus par Mohammed à cette occasion, pour justifier que le Musulman qui tombe en martyr soit enveloppé dans les vêtements qu'il portait en combattant et inhumé ainsi, sans que son cadavre soit lavé, ni que les prières habituelles soient dites : « Enroulez-les avec leurs blessures dans leurs vêtements et, au moment du Jugement dernier, lors de la résurrection, le sang sortira de leur veine du cou [...] leur haleine exhalera l'odeur du musc ${ }^{37}$ » Quant au chahîd lui-même, il est dispensé d'attendre le Jugement dernier (sa place est réservée) et il jouit immédiatement de ces vierges inaccessibles sur terre... A moins que ce ne soit - comme le suggère un érudit orientaliste allemand - qu'une erreur de traduction répétée sans cesse : il s'agirait alors de blanches nuées et donc plutôt de la pureté d'une extase due à la seule présence divine... Assez curieusement, nous trouvons peu de commentaires insistant sur la jouissance de l'absolu et de la plénitude divine, alors que les textes sont, sur ce point, exceptionnellement clairs: «Dis! Si l'ultime séjour pour vous seuls à l'exclusion des autres est auprès de Dieu, souhaitez la mort si vous êtes véridiques [...] ${ }^{38}$ ". Les mystiques veulent se fondre dans l'Un, certes, mais pas au prix de la mort volontaire. Celle-ci ne semble invoquée que dans le chiisme, or la plupart des « kamikazes » sont sunnites, ce qui n'est pas la moindre des contradictions. Le politique ou l'immanent l'emporteraient-ils sur le transcendant?

Là encore, l'explication psychanalytique paraît la plus pertinente pour tenter de comprendre et d'expliquer cet écart, dans la mesure où l'irruption de la transcendance constitue l'une des voies de sortie possibles du conflit externe entre l'idéal du moi collectif et celui du moi individuel, notamment en cas de tension et de culpabilité face à l'hégémonie coloniale allogène. C'est la mise en scène de la mort héroïque du moi idéal archaïque. On retrouve ce même mécanisme dans les suicides dits altruistes et probablement chez bon nombre de martyres de l'Histoire. En effet, si on applique la théorie de la pulsion de mort, celle-ci met en jeu de façon subtile la pulsion de vie en proposant une vie éternelle et la satisfaction libidinale, l'octroi de ces vierges pour l'éternité ${ }^{39}$.

Le premier cas de «nouveau combattant suicidaire» islamique de la période contemporaine ${ }^{40}$ est, sauf erreur, celui chanté par Fadwa Touqan, poétesse palestinienne qui, dans son recueil Al-layl wa al-fursan ${ }^{41}$, cite un combat, qui a eu lieu le 30 septembre 1967, durant lequel Mazin Abû Ghazalah se fait exploser avec une grenade au milieu des assaillants. La terre nourricière accueille le sacrifice comme la mère du chahîd l'accepte. Elle connaît son Coran : « Ne croyez pas que ceux qui sont tués en combattant dans la voie de Dieu sont morts; au contraire, ils vivent et sont nourris auprès de leur Seigneur ${ }^{42}$ ». Ce verset a toutefois donné lieu à plusieurs explications / commentaires différents: pour certains le chahîd est vivant, pour d'autres il est vivant au paradis alors qu'il était mort sur terre. 

saint Paul, annonciateur de toute la martyrophilie chrétienne : «Pour moi, la vie c'est le Christ, et mourir représente un gain : je préfère une mort qui conduit à la vie qu'une vie qui conduit à la mort ${ }^{43}$ ». Le lecteur attentif trouvera plusieurs citations en ce sens dans ses Epîtres. éternel plutôt que le séjour périssable, y compris dans des hadiths/dicts du Prophète honorant la "mère des martyrs ». Mais cette éducation/formation à base de hadiths, de citations du Coran, d'hagiographies apologétiques et de traditions populaires ne fonctionnerait pas aussi facilement, même en période de crise grave comme c'est le cas actuellement, si elle ne reposait pas sur des structures plus archétypales, ou tout du moins, ancrées dans une histoire longue.

Suicide polémique, sacrifice et violence

Il faut, pour prendre du recul sur cette violence inacceptable mais explicable, effectuer tout un parcours : de Gilbert Durand ${ }^{44}$, qui prétend que les structures anthropologiques de notre imaginaire constituent un socle certes oublié mais actuel, à Pierre Legendre ${ }^{45}$ qui ne cesse de dénoncer l'oubli de notre ontogénèse. Il nous faut relire encore Pierre Solié qui insiste sur le "sacrifice fondateur de civilisation et d'individuation ${ }^{46}$ " et risque une comparaison avec l'endo-sacrifice dans l'orgasme d'orgie, sans oublier Marie Balmary et son idée de sacrifice interdit ${ }^{47}$.

Aussi ne paraît-il pas possible d'éviter le rappel du mythe fondateur de notre civilisation: le meurtre du frère, meurtre de l'Autre si proche, mythe de la violence fondatrice bien plus éclairant que celui de l'hypothétique banquet au cours duquel, selon Freud, on consommait le père ${ }^{48}$. Osiris est assassiné et découpé en morceaux par son frère Horus, Caïn tue Abel ${ }^{49}$, Ismaël est banni au profit d'Isaac ${ }^{50}$, sans oublier Jacob et Esaü ${ }^{51}$, ni le sacrifice d'Abraham ${ }^{52}$, Moïse et l'Egyptien qu'il tue ${ }^{53}$, ๔edipe, Romulus et Remus et enfin Ali, calife et gendre du Prophète et l'un de ses fils assassinés. De tout cela ressort l'importance de la martyrologie/philie dans le chiisme manifestée lors de la « fête » d'Achoura. Mais on ne saurait oublier Martin Luther King, lui aussi assassiné et, bien entendu, le Christ crucifié comme holocauste suprême.

36 A travers tous ces mythes fondateurs, c'est bien la violence homicide qui constitue notre premier «être-ensemble». Le rapport au sens du sang ${ }^{54}$ est ainsi fondateur même dans l'holocauste ${ }^{55}$ christique consenti, illustrant la thèse du «bouc émissaire " de René Girard pour lequel la victime est nécessairement innocente ! Or, les Musulmans - dont on oublie trop souvent en Occident qu'ils sont les premières victimes massives du terrorisme dit "islamiste radical», mais aussi les derniers de «l'entre-nous originel » à pratiquer le sacrifice du mouton - ont gardé le même rapport au sang que les Juifs : circoncision, interdiction de consommer du sang et endogamie. l'égorgement rituel, est un acte propitiatoire, sanctifiant les acteurs et la victime. Il faut avoir vu ces enfants pataugeant dans le sang de la fête pendant que les pères remplissent leur devoir sacerdotal pour comprendre que le rapport au sang n'est pas le même que dans nos sociétés occidentales.

Dans la violence des guerres de libération nationale ou dans la violence conjugale, l'égorgement n'est pas vécu comme plus barbare que les bombardements de populations civiles par des armées étrangères, en vertu d'une différence culturelle dont 
nous devons tenir compte pour comprendre - à défaut d'excuser - certains actes révoltants à nos yeux.

Mais l'archéologie récente de la violence meurtrière imprègne aussi nos visions du monde de l'Autre. Elle ne date pas des «islamistes-terroristes-égorgeurs de femmes et d'enfants » et il ne s'agit pas simplement de vertige nihiliste ! L'Histoire, même récente, nous en offre une large palette: violence des rapports précoloniaux lorsque les Ottomans, puis les Turcs n'étaient guère tendres avec leurs coreligionnaires dominés ; violence des guerres de conquêtes coloniales où - après le scalp - on collectionnait les oreilles et les testicules portés triomphalement en collier dans les salons des capitales "civilisées"; violences des rapports coloniaux, sur lesquelles le psychiatre martiniquais Frantz Fanon a tout dit ${ }^{56}$; violences des guerres de décolonisation où chacune des parties a perdu son honneur dans la torture, l'assassinat, le viol et la mutilation systématiques; violences des régimes dictatoriaux de gauche ou progressistes-nationalistes, des généraux algériens, égyptiens, syriens, bien avant la violence « islamiste » ou encore celle des puritains d'Arabie Saoudite qui décapitent au sabre leurs délinquants supposés; violences, enfin, faites au peuple palestinien, qui n'est pas responsable de la Shoah mais qui subit depuis cinquante ans, outre la violence de l'armée israélienne, la concussion et l'autodestruction de ses propres dirigeants. Sans oublier le mépris des Etats arabes surarmés qui se contentent d'un soutien verbal et hypocrite à la "cause sacrée » quand ils ne manipulent pas eux aussi leurs propres sous-groupes concurrentiels palestiniens. Il faut avoir vu le cloaque de Gaza, les pères humiliés et l'arrogance des nouveaux riches pour comprendre l'influence exercée par certains groupes extrémistes sur la jeunesse palestinienne. Mais les pierres ne peuvent rien contre les chars ou les hélicoptères de combat. Encore moins contre le mépris et l'abandon des alliés « naturels ».

Jusqu'à une date récente, les assassinats, les tortures ou les morts au combat représentaient en quelque sorte la norme. Or, depuis deux décennies environ, nous avons vu apparaître dans l'aire arabo-islamique deux catégories fort différentes de nouveaux combattants suicidaires : les Palestiniens et Al-Qaïda. Le rapport de force dans la guerre asymétrique a changé et les puissants en sont étonnés : comment les barbares osent-ils violer nos lois de la guerre et venir « jusque dans nos bras égorger nos fils et nos compagnes »?

41 Le monde libre déclare alors la guerre au terrorisme fanatique islamique tandis que George W. Bush, sorte de "Bibleman » super-héros de l'Eglise évangélique ${ }^{57}$, veut extirper le Mal par la croisade et répond ainsi point par point au discours de Ben Laden évoquant le Taghut ${ }^{58}$, qui désigne, dans le Coran, le Mal au sens de « rebelle à Dieu ». De plus, pour mieux se faire comprendre, il affuble les Occidentaux du terme de " croisés », ces Salabiyyin honnis de tout Musulman bien né.

Alors la mort auto-administrée des «kamikazes »/chouhada devient une arme contre une culture basée sur le mépris et la mort de l'Autre, à l'intérieur comme à l'extérieur. Une réponse à la non-réponse $\mathrm{du}$ " nous » musulman et aussi une réponse à la théorie du « zéro mort » chez les boys qui crient « Maison, maison! » en détruisant celles des autres ${ }^{59}$ !

Mais les despotes arabes et tous les pillards de la rente pétrolière peuvent dormir tranquilles pendant encore quelques temps tandis que l'Occident médiatique se demande pourquoi les terroristes s'en prennent à lui... Il faut lire, parmi la douzaine de livres parus actuellement sous la plume de différents experts du renseignement, le 
surprenant ouvrage de Bauer et Raufer sur L'Enigme d'Al-Qaïda ${ }^{60}$ qui soutient que, pendant que la presse et les peuples sont "terrorisés " par les terroristes, les vraies affaires non contrôlées profitent à certains...

L'alliance entre les puritains américains et les puritains wahhabites comme éthique du capitalisme libéral mondialisé est l'une des grandes innovations de ce début de siècle, mais elle n'a pas commencé avec G.W. Bush, puisque Roosevelt, lui-même persuadé que Dieu l'avait missionné pour sauver le monde en y mettant de l'ordre ${ }^{61}$, s'est arrêté en Arabie Saoudite en rentrant de Yalta en 1945, hissant ainsi le royaume des Bani Sa'ud au rang de premier allié des Etats-Unis!

On est bien dans une nouvelle forme de géopolitique hégémonique, dans laquelle la manipulation de la religion masque des intérêts concrets et où ce sont les plus aliénés qui se salissent les mains dans le sang pour le compte et au bénéfice de leurs vrais ennemis. Comme cela serait complètement insupportable à tout être informé et doué de raison, seule l'intériorisation de la légitimité transcendantale nationaliste, communautariste et "religieuse » permet aux acteurs d'agir jusqu'au bout de leur logique interne: mourir pour le Royaume à venir car, pour qu'il advienne, il faut détruire ce monde impie!

\section{NOTES}

1.. Terme impropre, littéralement, comme renvoyant de manière spécifique à une tout autre civilisation (voir Pinguet M., La Mort volontaire au Japon, Paris, Gallimard, 1984). Comme tous les Occidentaux qui ont quelque difficulté à nommer le suicide de combat, nous aussi dirons bien « kamikazes » mais avec des guillemets.

2.. Bergeret J., La Violence et la Vie, Payot, 1994, La Pathologie narcissique, Paris, Dunod, 1996, La Psychologie pathologique, Paris, Masson, 1997, La Violence fondamentale, Paris, Dunod, 2000.

3.. Voir les Cahiers des lectures freudiennes, n²4, Paris, La Lysimaque, 1998.

4.. Republié dans Essais de psychanalyse, Payot, 1968, pp. 7-82 (coll. «Petite Bibliothèque Payot »).

5.. Concept largement étudié par Conrad Lorenz.

6.. Littéralement « chute des feuilles des arbres » en grec. Concept développé en 1975 par A.H. Willie et J.F.R. Kerr. Il existe des phénomènes de mort à l'intérieur même des mécanismes de la vie. Voir De la cellule à l'Homme: des morts programmées, Nice, Académie européenne interdisciplinaire des sciences Nice-Côte d'Azur, PUF, 2001.

7.. Terme d'origine japonaise utilisé par les médias bien qu'étant impropre en ce qui concerne les musulmans. Il signifie « le vent des dieux » ou « les dieux du vent », le caractère « kanji » pouvant se lire dans les deux sens. Durant l'opération dite « kamikaze tokubetsu kögekitai » («forces d'attaque spéciale du vent des dieux »), 2198 pilotes se sacrifièrent, 34 navires américains furent coulés et endommagés. 
8.. Référence à ceux qui, dans l'islam, ont le pouvoir de lier et de délier, d'organiser et de gérer le «pourchas » du Mal (comme disent les islamologues) et la commanderie du Bien.

9.. Bien entendu, l'essai majeur d'Emile Durkheim sur le suicide est toujours d'un grand secours : Le Suicide, Paris, PUF (coll. « Quadrige »), 11e édition, 2002.

10.. Voir en particulier La Violence et le Sacré, Paris, Grasset, 1972, Le Bouc émissaire, Paris, Grasset, 1982.

11.. J'ai déjà abordé cette aporie dans une première version de mon essai, Les Amants de l'Apocalypse, Paris, Editions de l'Aube, 2002, en rappelant qu'» apocalypse » signifie "révélation », en renvoyant également aux passionnants travaux de N. Kohn., auteur, entre autres, des Fanatiques de l'Apocalypse, Paris, Juillard, 1962 et de Cosmos, Chaos et le Monde, Paris, Allia, 2000.

12.. Voir l'excellent travail du Cercle de réflexion pour la psychanalyse en Algérie : "Algérie, années 90 : politique du meurtre. Pour une lecture freudienne de la crise algérienne ", Cahiers de lectures freudiennes $n^{\circ} 24$, op. cit.

13.. Coran III, 145 : «Il n'appartient [à aucun être vivant] à personne de mourir que par la permission de Dieu d'une prescription déterminée ».

14.. Coran II, 178-179 : « la loi du talion vous est prescrite à l'égard des tués : l'homme libre contre l'homme libre, l'esclave sauf atténuation de cette rigueur de faveur de qui quelque chose sera remis par son frère [...]».

15.. Coran IV, 32 : «Quiconque a tué un homme sans que celui-ci ait tué ou semé la discorde dans le pays sera considéré comme s'il avait tué l'humanité tout entière [...] ».

16.. Le meurtre du prince impie est une nécessité religieuse, tant chez saint Thomas d'Aquin que chez Ibn Taymiyya, auteurs respectivement de Somme théologique (ouvrage interrompu par la mort de son auteur en 1274) et de Kitab al-siyasa al-sha'riya [Traité de politique islamique], 1311.

17.. Par exemple en Coran VIII, 17.

18.. Littéralement : « désordre ».

19.. Voir le film de Simone Bitton Le Mur, France, Israël, 2004, qui fait bien comprendre ce qu'est l'amixia, le non-mélange du « pur » et de l'» impur ».

20.. Shamir Y., Check-points, (titre original : Machssomim), Israël, 2003.

21.. Voir Hadj-Mouri A., Quelques considérations psychanalytiques sur le discours de l'intégrisme musulman en Algérie, Association Patou, n²72, avril 1999, Lille, Bibliothèque freudienne de Lille.

22.. Pour les lecteurs qui ont le goût de l'exégèse, voir entre autres Coran III, 145, Coran VIII, 17, Coran IX, 111, Coran XLII, 42, etc.

23.. Charnay J.-P., Principes de stratégie arabe, Paris, L'Herne, 2003. La " guerre juste » du jihâd implique que la Maison de l'islam ("Dar al-islam »), soit attaquée, occupée ou encore mise en danger de l'intérieur par les Musulmans eux-mêmes (Coran II, 216, VIII, 39 , IX, 29 et 41).

24.. Cohen D., « Terrorisme : la pauvreté n'est pas coupable », Le Monde, 7 octobre 2002. Enquête de l'Agence palestinienne Center for Policy and Survey Research, confirmant les résultats d'une enquête sur l'IRA, les Brigades rouge, la Bande à Baader, l'Armée rouge japonaise : les deux tiers des auteurs des attentats ont fait des études supérieures et émanent de milieux aisés.

25.. A ma connaissance, deux cas ont été signalés en Palestine/Israël : l'une était religieuse et célibataire, l'autre laïque et mère de famille. Un responsable du Hamas, Sami Abu Zuhri, déclare cependant : «Il y a des centaines de femmes attaquantes martyres, 
qui affluent en masse et réclament de participer aux opérations martyres. C'est un phénomène unique en son genre, qui reflète la force de l'esprit du Jihad au sein de ce peuple [...]. Ce peuple manifeste avec force son adhésion à l'option du martyre, tout particulièrement à la lumière des exemples de femmes auteurs d'attaques martyres. La résistance palestinienne, parfois, utilise expressément des femmes pour certaines opérations que les hommes ne peuvent mener, particulièrement dans les zones de haute sécurité que les Moudjahidin masculins ne peuvent pas facilement atteindre. C'est pourquoi la femme palestinienne joue un rôle important dans la résistance palestinienne et peut parfois jouer des rôles que les jeunes moudjahidin ne peuvent remplir. » Al-Majd TV (EAU), 13 juin 2004. Clip \#117 pouvant être consulté avec les réserves d'usage sur http://memritv.org/archives.asp.

26.. Pluriel de chahid.

27.. Coran IX, 111.

28. Les Israeliens juifs orthodoxes ramassent les débris des corps éclatés par les attentats car, pour que la résurrection finale soit possible, il faut enterrer un cadavre « entier».

29.. L'explication des « couches » trouvés sur certains corps est sans doute là, mais aussi pour conserver intactes les parties sexuelles.

30.. Coran XXII, 5.

31.. Coran XXXII, 11.

32.. Chargé du décompte des bonnes et mauvaises actions du défunt au jour $\mathrm{du}$ Jugement.

33.. Respectivement anges annonciateurs de la mort et du Jugement dernier.

34.. Auteur de al-ahkâm al-sultaniya [les statuts gouvernementaux].

35. Victoire remportée par Muhammad en 623 sur une caravane de Qorayshites qui revenait de Syrie.

36.. Les « vies hagiographiques voire apologétiques du Prophète ».

37.. Cité par Mawardi dans Arkan al-sultannya, trad. par E. Fagnan, Alger, Jourdan, 1915, p. 85.

38.. Coran. II, 94 et LXII, 6.

39.. Le comparatisme démontre néanmoins que d'autres types de « kamikazes » peuvent avoir d'autres motivations! Mais les cas récents ne sont pas très nombreux, mises à part des actions « lourdes » des Tigres tamouls et quelques cas en partie comparables signalés au Liban et peut-être en Russie/Tchétchénie où les «veuves noires » semblent jouer un rôle dramatique.

40. Il serait intéressant d'étudier la prégnance du mythe de Massada et des Zélotes sur les jeunes recrues israéliennes. La Bible nous donne au moins un cas : Samson secouant les piliers du temple périt en tuant tout le monde avec lui. Voir Dufour Y.R., Le Suicide dans les textes de la tradition juive, www.modia.org.

41.. La Nuit et les Cavaliers. Sur Internet : fr.wikiquote.org/wiki/Fadwa_Touquan.

42.. Coran III, 163.

43. Saint Paul, Actes, 20,24.21,13. Epître aux Romains, 8,3. et aussi Actes, 6,10-11,14,8

44.. Gilbert Durand est le fondateur du Centre de recherche sur l'imaginaire. Il est l'auteur, entre autres, de L'Anthropologie de l'imaginaire, Paris, Bordas, 1983.

45.. Les Leçons, Paris, Fayard (surtout la VIIe « le désir politique de Dieu » et la VIIIe « le crime du caporal Lortie »), mais aussi « Dieu au miroir », L'Amour du censeur, Paris, Seuil, 1974 et surtout une conférence-débat à Toulouse (inédite) donnée au GREP, le 23 janvier 1999. 
46.. Solié P., Le Sacrifice: fondateur de civilisation et d'individuation, Paris, Albin Michel, 1988.

47.. Balmary M., Le Sacrifice interdit, Paris, Grasset, 1986.

48.. On sait la réticence de J.P. Vernant et de R. Girard envers cette thèse.

49.. Genèse 4, 1-16.

50.. Genèse 21, 8-19.

51.. Genèse 27.

52.. Genèse 22. Voir le texte de Louis Fedi « Abraham », aux éditions Limes, 2004.

53.. Exode 2, 11-14.

54.. Voir Dieterlen G., qui, dans ses travaux sur les dogons a montré que le sang véhiculait la force vitale (libido ou eros), et pas seulement l'impureté et la souillure retenues par certains ethnologues et affirmées par certains clercs monothéistes! Voir Journet 0. , «Le sacrifice, rites d'eau et de sang pour d'autres dieux ", Lumières et vie, n¹46, Lyon, 1980.

55.. Dans le judaïsme depuis le sacrifice du « premier né » jusqu'aux holocaustes d'animaux : la description de la Olah fait l'objet de plusieurs paragraphes comme « II Rois ", $3: 26-27$, Nombres, 21 et suivantes, Lévitique, 23, etc.

56.. Fanon F., Les Damnés de la terre, Paris, Maspero, 1961.

57.. Voir le film de Roney Aronson, G.W. Bush sous l'emprise de Dieu, Etats-Unis, 2004.

58.. Voir l'excellent article de Slimane Zeghidour sur ce sujet dans Télérama, 20 octobre 2004, accompagné d'une bibliographie exhaustive récente sur les fondamentalistes américains, p. 16.

59.. Nous faisons ici référence à l'assertion, au « slogan » pourrait-on dire, des chefs d'Etat américains, qu'on retrouve dans les News et dans une grande majorité des films de guerre : «Boys ! On rentre à la maison!».

60.. Bauer A., Raufer X., L'Enigme d'Al-Qaïda, Paris, JC Lattès, 2005.

61.. L'éthique protestante et l'esprit du capitalisme ont parfaitement été intériorisés par les Bani Sa'ud et autres « princes » arabes descendants directs des tribus de pillards bédouins du désert.

\section{RÉSUMÉS}

La presse occidentale utilise le terme de « kamikaze » pour désigner les combattants suicidaires. Eux-mêmes emploient un tout autre vocabulaire qui renvoie à la culture islamique et au statut de la mort, du suicide, du martyr dans l'historiographie utilisée par les « Islamistes ». Ce dernier terme est lui aussi polysémique et nous devons distinguer, pour expliquer les opérations suicidaires, des groupes dont la référence est parfois commune mais les buts complètement différents : le Hamas n'est pas le GIA algérien ni le Hezbollah libanais et encore moins Al-Qaïda. La méconnaissance est au principe du traitement de l'information. Notre devoir est d'essayer d'expliquer et de comprendre même l'inadmissible.

Western media often use the word "kamikaze" to designate suicidal combatants. The latter however use a quite different vocabulary referring to the Islamic culture and the status of death, suicide and martyrdom within the historiography used by "Islamists". The latter word is also 
polysemous and in order to explain suicidal operations we have first to make distinctions between groups having different aims but the references of which might be common: the Hamas is neither the Algerian GIA, the Lebanese Hizbollah, and even less Al-Qaeda. Misperceptions of these differences often characterise the analysis of these groups. Our duty is therefore to try to explain and understand the unacceptable.

INDEX

Index géographique : Moyen-Orient, Palestine

Mots-clés : kamikaze (missions suicide), religion

Thèmes : Hamas (Palestine)

\section{AUTEUR}

\section{BRUNO ETIENNE}

Bruno Etienne est membre de l'Institut universitaire de France et directeur de l'Observatoire du religieux à l'Institut d'études politiques d'Aix-en-Provence. 